University of Warwick institutional repository: http://go.warwick.ac.uk/wrap This paper is made available online in accordance with publisher policies. Please scroll down to view the document itself. Please refer to the repository record for this item and our policy information available from the repository home page for further information.

To see the final version of this paper please visit the publisher's website. Access to the published version may require a subscription.

Author(s): BAS LEMMENS and MICHAEL SCHEUTZOW

Article Title: On the dynamics of sup-norm non-expansive maps

Year of publication: 2005

Link to published

version: http://dx.doi.org/10.1017/S0143385704000665

Publisher statement: None 


\title{
On the dynamics of sup-norm non-expansive maps
}

\author{
BAS LEMMENS $\dagger$ and MICHAEL SCHEUTZOW $\ddagger$ \\ $\dagger$ Mathematics Institute, Warwick University, Coventry CV4 7AL, UK \\ (e-mail:lemmens@maths.warwick.ac.uk) \\ $\ddagger$ Institut für Mathematik, MA 7-5, Technische Universität Berlin, \\ Straße des 17. Juni 136, D-10623 Berlin, Germany \\ (e-mail:ms@math.tu-berlin.de)
}

(Received 12 September 2003 and accepted in final form 30 June 2004)

\begin{abstract}
We present several results for the periods of periodic points of sup-norm nonexpansive maps. In particular, we show that the period of each periodic point of a sup-norm non-expansive map $f: M \rightarrow M$, where $M \subset \mathbb{R}^{n}$, is at $\operatorname{most}_{\max _{k}} 2^{k}\left(\begin{array}{l}n \\ k\end{array}\right)$. This upper bound is smaller than $3^{n}$ and improves the previously known bounds. Further, we consider a special class of sup-norm non-expansive maps, namely topical functions. For topical functions $f: \mathbb{R}^{n} \rightarrow \mathbb{R}^{n}$ Gunawardena and Sparrow have conjectured that the optimal upper bound for the periods of periodic points is $\left(\begin{array}{c}n \\ \lfloor n / 2\rfloor\end{array}\right)$. We give a proof of this conjecture. To obtain the results we use combinatorial and geometric arguments. In particular, we analyse the cardinality of anti-chains in certain partially ordered sets.
\end{abstract}

\section{Introduction}

In this paper we are concerned with the dynamics of sup-norm non-expansive maps $f: M \rightarrow M$, where $M \subset \mathbb{R}^{n}$. A characteristic property of the iterative behaviour of sup-norm non-expansive maps is that every bounded orbit converges to a periodic orbit. Moreover, there exists an upper bound for the possible periods of periodic points of supnorm non-expansive maps that only depends on the dimension of the ambient space. It is, therefore, an interesting problem to determine the optimal upper bound for the periods of periodic points of sup-norm non-expansive maps. This problem has been considered in $[4,14-16,18,25]$, and Nussbaum $[18]$ has conjectured that $2^{n}$ is the optimal upper bound. At present, however, the conjecture is proved only for $n \leq 3$ (see [15]).

The main goal of the paper is to present two results concerning the periodic points of sup-norm non-expansive maps. As a first result, we show that the period of each periodic point of a sup-norm non-expansive map $f: M \rightarrow M$, where $M \subset \mathbb{R}^{n}$, is at $\operatorname{most}_{\max } 2^{k}\left(\begin{array}{l}n \\ k\end{array}\right)$. This upper bound is smaller than $3^{n}$ and is a considerable improvement of the previously best-known estimate $n ! 2^{n}$ by Martus [16]. 
It turns out that the ideas for proving our estimate can be used to analyse the periods of periodic points of topical functions. Topical functions are maps $f: \mathbb{R}^{n} \rightarrow \mathbb{R}^{n}$ that are order-preserving and additively homogeneous. They are sup-norm non-expansive by a result of Crandall and Tartar [7], and they appear in a variety of applications, such as the analysis of discrete event systems $[\mathbf{3}, \mathbf{5}, \mathbf{9}, \mathbf{1 0}]$, optimal control theory [2] and nonlinear Perron-Frobenius theory $[\mathbf{8}, \mathbf{1 9}, \mathbf{2 0}]$. As a second result, we prove a conjecture of Gunawardena and Sparrow (see [9, §4.3] and [10, §2.1.3]), which asserts that the optimal upper bound for the periods of periodic points of topical functions $f: \mathbb{R}^{n} \rightarrow \mathbb{R}^{n}$ is $\left(\begin{array}{c}n \\ \lfloor n / 2\rfloor\end{array}\right)$.

To obtain the results we analyse transitive actions of Abelian groups of sup-norm isometries. As explained in the next section, this is a more general setting than is required. In fact, in order to obtain an optimal upper bound for the periods of periodic points of sup-norm non-expansive maps, one could consider transitive actions of cyclic groups of sup-norm isometries. But, as yet, we do not know how to (or if it is necessary to) exploit this additional assumption.

The dynamics of non-expansive maps has been studied for various norms on $\mathbb{R}^{n}$. In particular, it is known that if the unit ball of the norm is a polyhedron, then every bounded orbit of a non-expansive map converges to a periodic orbit (see [18] or [26]). This is an immediate consequence of the result for the sup-norm, because any finitedimensional polyhedrally normed space can be isometrically embedded into $\mathbb{R}^{n}$ with the sup-norm, as long as $n$ is sufficiently large. Another interesting polyhedral norm on $\mathbb{R}^{n}$, besides the sup-norm, is the $\ell_{1}$-norm. There exist many detailed results for the possible periods of periodic points of $\ell_{1}$-norm non-expansive maps and we refer the reader to $[\mathbf{1 , 1 2}, \mathbf{1 3}, \mathbf{1 7}, \mathbf{2 1 - 2 4}]$ for further information.

In addition to the introduction the paper contains four sections. In $\$ 2$ we collect several definitions and results for sup-norm non-expansive maps. Furthermore, we formulate a theorem from which it follows that the period of each periodic point of a sup-norm nonexpansive map $f: M \rightarrow M$, where $M \subset \mathbb{R}^{n}$, is at $\operatorname{most}_{\max _{k}} 2^{k}\left(\begin{array}{c}n \\ k\end{array}\right)$. Subsequently, we prove this more general theorem in $\S 3$. Section 4 contains several definitions and results concerning topical functions. In addition, we state a theorem that implies that the period of each periodic point of a topical function $f: \mathbb{R}^{n} \rightarrow \mathbb{R}^{n}$ is at most $\left(\begin{array}{c}n \\ \lfloor n / 2\rfloor\end{array}\right)$. Section 5 is devoted to a proof of this theorem. We conclude $\$ 5$ with several remarks. In particular, we explain a consequence of our results for the dynamics of certain cone maps.

\section{Preliminaries}

Let $\mathbb{R}^{n}$ be equipped with the sup-norm, given by $\|x\|_{\infty}=\max _{i}\left|x_{i}\right|$ for $x=\left(x_{1}, \ldots, x_{n}\right)$ in $\mathbb{R}^{n}$. A map $f: M \rightarrow M$, where $M \subset \mathbb{R}^{n}$, is called sup-norm non-expansive if $\|f(x)-f(y)\|_{\infty} \leq\|x-y\|_{\infty}$ for all $x, y \in M$. It is said to be a sup-norm isometry if equality holds for all $x, y \in M$. A group $\Gamma$ of sup-norm isometries $g: X \rightarrow X$, where $X \subset \mathbb{R}^{n}$, is said to act transitively on $X$ if for each $x, y \in X$ there exists $g \in \Gamma$ such that $g(x)=y$.

A point $x \in M$ is called a periodic point of $f: M \rightarrow M$ if there exists an integer $p \geq 1$ such that $f^{p}(x)=x$. The minimal such $p \geq 1$ is called the period of $x$ under $f$. Transitive actions of groups of isometries are intimately connected with 
periodic points of non-expansive maps, as we shall explain now. Let $x \in M$ be a periodic point of $f: M \rightarrow M$ with period $p$ and let $X$ be the orbit of $x$ under $f$; so, $X=\left\{f^{k}(x) \mid 0 \leq k<p\right\}$. If $f$ is sup-norm non-expansive, then the restriction of $f$ to $X$ is a sup-norm isometry that maps $X$ onto itself. Moreover, if we put $g=f_{\mid X}$, then $\left\{g^{k}: X \rightarrow X \mid 0 \leq k<p\right\}$ is a cyclic group of sup-norm isometries that acts transitively on $X$. Thus, to prove that the period of each periodic point of a sup-norm non-expansive map $f: M \rightarrow M$, where $M \subset \mathbb{R}^{n}$ is at $\operatorname{most}_{\max _{k}} 2^{k}\left(\begin{array}{l}n \\ k\end{array}\right)$, it suffices to show that each finite set $X$ in $\mathbb{R}^{n}$, with a transitive Abelian group of sup-norm isometries, has at $\operatorname{most}_{\max _{k}} 2^{k}\left(\begin{array}{l}n \\ k\end{array}\right)$ elements. In the paper we take this approach. In fact, to estimate the periods of periodic points of sup-norm non-expansive maps, we prove the following theorem in $\S 3$.

THEOREM 2.1. If $X$ is a finite set in $\mathbb{R}^{n}$ on which an Abelian group of sup-norm isometries acts transitively, then $|X| \leq \max _{k} 2^{k}\left(\begin{array}{c}n \\ k\end{array}\right)$.

Let us first discuss some preliminary results. We would like to mention that several of these results can be found in $[\mathbf{1 5}, \S 2]$. For the reader's convenience we prove them here once more.

Actions of Abelian groups of isometries enjoy the following useful property (compare [15, Lemma 2.3]).

LEMMA 2.2. If $X$ is a set in $\mathbb{R}^{n}$ on which an Abelian group $\Gamma$ of sup-norm isometries acts transitively, then for each $f \in \Gamma$ there exists a constant $d(f)$ such that $\|x-f(x)\|_{\infty}=$ $d(f)$ for all $x \in X$.

Proof. Since $\Gamma$ is an Abelian group of sup-norm isometries we have that

$$
\|x-f(x)\|_{\infty}=\|g(x)-g(f(x))\|_{\infty}=\|g(x)-f(g(x))\|_{\infty} \quad \text { for all } g \in \Gamma .
$$

As $\Gamma$ acts transitively on $X$, it follows that $\|x-f(x)\|_{\infty}$ is the same for all $x \in X$, which completes the proof.

A sequence of points $x^{1}, x^{2}, \ldots, x^{m}$ in $\mathbb{R}^{n}$ is called an additive chain if

$$
\left\|x^{1}-x^{m}\right\|_{\infty}=\sum_{k=1}^{m-1}\left\|x^{k}-x^{k+1}\right\|_{\infty} .
$$

We say that it has length $m$ if it consists of $m$ distinct points. Lemma 2.2 has the following corollary for additive chains (compare [15, Proposition 2.1]).

Corollary 2.3. Suppose that $X$ is a finite set in $\mathbb{R}^{n}$ on which an Abelian group $\Gamma$ of sup-norm isometries acts transitively. If $x^{1}, x^{2}, \ldots, x^{m}$ is an additive chain in $X$ of length $m \geq 2$, and for $1 \leq k<m$ the map $f_{k} \in \Gamma$ maps $x^{k}$ to $x^{k+1}$, then for each permutation $\pi$ on $\{1, \ldots, m-1\}$ and for each $x \in X$ the sequence

$$
x, f_{\pi(1)}(x), \ldots, f_{\pi(m-1)} \circ \cdots \circ f_{\pi(1)}(x)
$$

is an additive chain of length $m$. 
Proof. Put $f=f_{m-1} \circ \cdots \circ f_{2} \circ f_{1}$ and observe that, as $\Gamma$ is Abelian, $f=f_{\pi(m-1)} \circ \cdots \circ$ $f_{\pi(2)} \circ f_{\pi(1)}$. For simplicity, we write $z^{k}=f_{\pi(k-1)} \circ \cdots \circ f_{\pi(1)}(x)$ for $2 \leq k \leq m$ and $z^{1}=x$. Using Lemma 2.2 we obtain the equalities

$$
\begin{aligned}
\left\|z^{1}-z^{m}\right\|_{\infty} & =d(f)=\left\|x^{1}-x^{m}\right\|_{\infty}=\sum_{k=1}^{m-1}\left\|x^{k+1}-x^{k}\right\|_{\infty}=\sum_{k=1}^{m-1} d\left(f_{k}\right) \\
& =\sum_{k=1}^{m-1} d\left(f_{\pi(k)}\right)=\sum_{k=1}^{m-1}\left\|z^{k+1}-z^{k}\right\|_{\infty} .
\end{aligned}
$$

Thus, $z^{1}, z^{2}, \ldots, z^{m}$ is an additive chain of length $m$, and this proves the corollary.

A useful idea in this exposition is the notion of an extreme pair. A pair of distinct points $\{x, y\}$ in $X$ is said to be an extreme pair in $X$ if there exists no $z \in X$ such that $z, x, y$ or $x, y, z$ is an additive chain of length 3 . Extreme pairs have the following property.

LEMMA 2.4. Suppose that $X$ is a finite set in $\mathbb{R}^{n}$ on which an Abelian group $\Gamma$ of supnorm isometries acts transitively. Then $\{x, y\}$ is an extreme pair in $X$ if and only if there exists no $z \in X$ such that $x, y, z$ is an additive chain of length 3.

Proof. It is clear from the definition that if $\{x, y\}$ is an extreme pair in $X$, then there exists no $z \in X$ such that $x, y, z$ is an additive chain of length 3 . To prove the other implication, we assume that $\{x, y\}$ is not an extreme pair in $X$. Then, there exists $z \in X$ such that $z, x, y$ or $x, y, z$ is an additive chain of length 3 . If $x, y, z$ is an additive chain of length 3 , we are done. On the other hand, if $z, x, y$ is an additive chain of length 3 , then we can use Corollary 2.3 to see that $x, y, f(y)$ is an additive chain of length 3 , where $f \in \Gamma$ is such that $f(z)=x$. This proves the lemma.

Further, we need to define a partial ordering $\preceq$ on $\{0,1,2\}^{n}$ by

$$
a \preceq b \text { if } a_{i}=b_{i} \text { for all } i \text { for which } b_{i} \in\{0,1\} .
$$

It is easy to verify that $\preceq$ is reflexive, anti-symmetric and transitive. A subset $\mathcal{A}$ of the partially ordered set $\left(\{0,1,2\}^{n}, \preceq\right)$ is called an anti-chain if there exist no distinct $a, b \in \mathcal{A}$ with $a \preceq b$. Using a standard method in extremal set theory, called the LYM technique [6, Ch. 7], we obtain the following upper bound for the cardinality of anti-chains in $\left(\{0,1,2\}^{n}, \preceq\right)$.

Proposition 2.5. If $\mathcal{A}$ is an anti-chain in $\left(\{0,1,2\}^{n}, \preceq\right)$, then

$$
|\mathcal{A}| \leq \max _{k} 2^{k}\left(\begin{array}{l}
n \\
k
\end{array}\right) \text {. }
$$

Proof. Consider chains $a^{0} \preceq a^{1} \preceq \cdots \preceq a^{n}$ in $\left(\{0,1,2\}^{n}, \preceq\right)$ consisting of $n+1$ distinct elements. There are $2^{n} n$ ! such chains. Indeed, to obtain such a chain one has to select a point $a^{0}$ with no coordinates equal to 2 . There are $2^{n}$ possibilities. Subsequently, one changes one by one the coordinates of $a^{0}$ to 2 . There are $n$ ! ways to do this. Thus, in total we have $2^{n} n$ ! such chains.

Now, consider an element $a$ in $\mathcal{A}$ with $k$ coordinates equal to 2, and we ask ourselves how many of the chains $a^{0} \preceq a^{1} \preceq \cdots \preceq a^{n}$ contain $a$. Clearly, there are $2^{k} k$ ! chains 
$a^{0} \preceq \cdots \preceq a^{k-1} \preceq a$, with $k+1$ distinct elements, and each of these can be extended in $(n-k)$ ! ways. Thus, there are $2^{k} k$ ! $(n-k)$ ! such chains.

To finish the argument, let $m_{k}$ be the number of elements in $\mathcal{A}$ with $k$ coordinates equal to 2. Obviously, $|\mathcal{A}|=\sum_{k=0}^{n} m_{k}$. As $\mathcal{A}$ is an anti-chain, each of the chains $a^{0} \preceq a^{1} \preceq \cdots \preceq a^{n}$ contains at most one element of $\mathcal{A}$. Therefore,

$$
\sum_{k=0}^{n} m_{k} 2^{k} k !(n-k) ! \leq 2^{n} n !
$$

so that

$$
\sum_{k=0}^{n} m_{k} 2^{k-n}\left(\begin{array}{l}
n \\
k
\end{array}\right)^{-1} \leq 1
$$

From this, we deduce that

$$
|\mathcal{A}|=\sum_{k=0}^{n} m_{k} \leq \max _{k} 2^{n-k}\left(\begin{array}{l}
n \\
k
\end{array}\right)=\max _{k} 2^{k}\left(\begin{array}{l}
n \\
k
\end{array}\right) .
$$

The upper bound in Proposition 2.5 is sharp. Indeed, the collection of points in $\left(\{0,1,2\}^{n}, \preceq\right)$ with $n-k$ coordinates equal to 2 is an anti-chain that contains $2^{k}\left(\begin{array}{c}n \\ n-k\end{array}\right)=$ $2^{k}\left(\begin{array}{l}n \\ k\end{array}\right)$ elements. From this remark, it follows directly that $\max _{k} 2^{k}\left(\begin{array}{l}n \\ k\end{array}\right)<3^{n}$. In fact, one can use Stirling's formula and some analysis to show that

$$
\max _{k} 2^{k}\left(\begin{array}{l}
n \\
k
\end{array}\right)=\gamma(n) 3^{n} / \sqrt{n}, \quad \text { where } 0<\delta_{1} \leq \gamma(n) \leq \delta_{2}<\infty \text { for all } n .
$$

\section{Proof of the first main result}

Proof of Theorem 2.1. Let $X$ be a finite set in $\mathbb{R}^{n}$ on which an Abelian group $\Gamma$ of sup-norm isometries acts transitively. Define $c: X \rightarrow\{0,1,2\}^{n}$ by

$$
c(x)_{i}= \begin{cases}0 \quad & \text { if there exists } y \in X \text { with }\{x, y\} \text { an extreme pair in } X \\ & \text { and }\|x-y\|_{\infty}=y_{i}-x_{i}, \\ 1 \quad \text { if there exists } y \in X \text { with }\{x, y\} \text { an extreme pair in } X \\ \text { and }\|x-y\|_{\infty}=x_{i}-y_{i}, \\ 2 \quad \text { otherwise, }\end{cases}
$$

for all $1 \leq i \leq n$ and $x \in X$.

We remark that $c(x)$ is well defined, because $c(x)_{i}=0$ and $c(x)_{i}=1$ implies that there exist $y, z \in X$ such that $\{x, y\}$ and $\{x, z\}$ are extreme pairs in $X$ with $\|x-y\|_{\infty}=y_{i}-x_{i}$ and $\|x-z\|_{\infty}=x_{i}-z_{i}$. This implies that

$$
\|y-z\|_{\infty} \leq\|y-x\|_{\infty}+\|x-z\|_{\infty}=y_{i}-x_{i}+x_{i}-z_{i}=y_{i}-z_{i} \leq\|y-z\|_{\infty},
$$

so that $z, x, y$ is an additive chain of length 3 . This, however, contradicts the fact that $\{x, y\}$ is an extreme pair in $X$.

By Proposition 2.5, it suffices to show that $c(y) \npreceq c(x)$ for all $x \neq y$ in $X$. To see this, let $\mathcal{F}$ be the collection of all additive chains $z^{1}, z^{2}, \ldots, z^{r}$ in $X$ such that $z^{1}=x$ and $z^{2}=y$. 
Now, consider an additive chain $z^{1}, z^{2}, \ldots, z^{m}$ of maximal length $m$ in $\mathcal{F}$. We claim that $\left\{z^{1}, z^{m}\right\}$ is an extreme pair in $X$. Indeed, there exists no $u \in X$ such that $z^{1}, z^{m}, u$ is an additive chain of length 3 , as $m$ is maximal. Therefore, it follows from Lemma 2.4 that $\left\{z^{1}, z^{m}\right\}$ is an extreme pair in $X$.

We observe that there exists $1 \leq i \leq n$ such that either $\left\|z^{1}-z^{m}\right\|_{\infty}=z_{i}^{m}-z_{i}^{1}$ or $\left\|z^{1}-z^{m}\right\|_{\infty}=z_{i}^{1}-z_{i}^{m}$. In the first case, $c(x)_{i}=0$ by definition, and we now show that $c(y)_{i} \neq 0$. If $c(y)_{i}=0$, then there exists $u \in X$ such that $\{y, u\}$ is an extreme pair in $X$ with $\|y-u\|_{\infty}=u_{i}-y_{i}$. As $z^{1}, z^{2}, \ldots, z^{m}$ is an additive chain and $\left\|z^{1}-z^{m}\right\|_{\infty}=z_{i}^{m}-z_{i}^{1}$, we have that

$$
\left\|z^{1}-z^{m}\right\|_{\infty}=z_{i}^{m}-z_{i}^{1}=\sum_{k=1}^{m-1} z_{i}^{k+1}-z_{i}^{k} \leq \sum_{k=1}^{m-1}\left\|z^{k+1}-z^{k}\right\|_{\infty}=\left\|z^{1}-z^{m}\right\|_{\infty} .
$$

This implies that $\left\|z^{k+1}-z^{k}\right\|_{\infty}=z_{i}^{k+1}-z_{i}^{k}$ for all $1 \leq k<m$, so that $\|x-y\|_{\infty}=y_{i}-x_{i}$. Hence, $x, y, u$ is an additive chain of length 3 , which contradicts the fact that $\{y, u\}$ is an extreme pair in $X$. Thus, we conclude that $c(y)_{i} \neq 0$ and hence $c(y) \npreceq c(x)$.

Similarly, if $\left\|z^{1}-z^{m}\right\|_{\infty}=z_{i}^{1}-z_{i}^{m}$, then by definition $c(x)_{i}=1$, but $c(y)_{i} \neq 1$. Indeed, if $c(y)_{i}=1$, then there exists $v \in X$ such that $\{y, v\}$ is an extreme pair in $X$ and $\|y-v\|_{\infty}=y_{i}-v_{i}$. Again, as $z^{1}, z^{2}, \ldots, z^{m}$ is an additive chain and $\left\|z^{1}-z^{m}\right\|_{\infty}=z_{i}^{1}-z_{i}^{m}$, it follows that $\|x-y\|_{\infty}=x_{i}-y_{i}$. This implies that $x, y, v$ is an additive chain of length 3 , which contradicts the fact that $\{y, v\}$ is an extreme pair. Thus, $c(y)_{i} \neq 1$ in this case and hence $c(y) \npreceq c(x)$. This completes the proof of the theorem.

\section{Topical functions}

Before we give the definition of a topical function we introduce some notation. On $\mathbb{R}^{n}$ we let $\leq$ be a partial ordering, given by $x \leq y$ if $x_{i} \leq y_{i}$ for all $1 \leq i \leq n$. Two elements $x$ and $y$ in $\mathbb{R}^{n}$ are said to be comparable if $x \leq y$ or $y \leq x$. A map $f: \mathbb{R}^{n} \rightarrow \mathbb{R}^{n}$ is called order-preserving if for each $x, y \in \mathbb{R}^{n}$ with $x \leq y$ we have that $f(x) \leq f(y)$. It is said to be additively homogeneous if $f(x+\lambda \mathbf{1})=f(x)+\lambda \mathbf{1}$ for all $x \in \mathbb{R}^{n}$ and $\lambda \in \mathbb{R}$. (Here $\mathbf{1}$ denotes the vector with all coordinates unity.) A topical function is a map $f: \mathbb{R}^{n} \rightarrow \mathbb{R}^{n}$ that is order-preserving and additively homogeneous.

Crandall and Tartar [7] have proved that every topical function is sup-norm nonexpansive. One can deduce this result from a useful observation of Gunawardena and Keane [11]. To formulate the observation we first need to introduce the function $t: \mathbb{R}^{n} \rightarrow \mathbb{R}$, given by $t(x)=\max _{i} x_{i}$ for all $x \in \mathbb{R}^{n}$.

Proposition 4.1. A map $f: \mathbb{R}^{n} \rightarrow \mathbb{R}^{n}$ is a topical function if and only if

$$
t(f(x)-f(y)) \leq t(x-y) \text { for all } x, y \in \mathbb{R}^{n} .
$$

Proof. Let $f: \mathbb{R}^{n} \rightarrow \mathbb{R}^{n}$ be a topical function and $x, y \in \mathbb{R}^{n}$. Obviously, $x \leq y+t(x-y) \mathbf{1}$, so that $f(x) \leq f(y)+t(x-y) \mathbf{1}$. This implies that $t(f(x)-f(y)) \leq t(x-y)$. To see the other implication, assume that $x \leq y$. Then $t(x-y) \leq 0$, so that $t(f(x)-f(y)) \leq 0$ and hence $f(x) \leq f(y)$. To show that $f$ is additively homogeneous, put $y=x+\lambda \mathbf{1}$. Clearly, $t(y-x)=\lambda$ and $t(x-y)=-\lambda$, which gives $t(f(y)-f(x)) \leq \lambda$ and $t(f(x)-f(y)) \leq-\lambda$. Thus, $\lambda \mathbf{1} \leq f(y)-f(x) \leq \lambda \mathbf{1}$, and this completes the proof. 
As $\|x\|_{\infty}=\max \{t(x), t(-x)\}$, Proposition 4.1 immediately implies that every topical function is sup-norm non-expansive.

The function $t$ is not a norm; it is, for instance, not symmetric. Nevertheless, we say that $f: M \rightarrow M$, where $M \subset \mathbb{R}^{n}$, is $t$-non-expansive if $t(f(x)-f(y)) \leq t(x-y)$ for all $x, y \in M$. Further, we call $f: M \rightarrow M$ a $t$-isometry if equality holds for all $x, y \in M$. We observe that if $x \in \mathbb{R}^{n}$ is a periodic point of a topical function $f: \mathbb{R}^{n} \rightarrow \mathbb{R}^{n}$ with period $p$, then the orbit of $x$, given by $X=\left\{f^{k}(x): 0 \leq k<p\right\}$, contains no comparable elements, as $f$ is order-preserving. Moreover, it follows from Proposition 4.1 that on $X$ a cyclic group of $t$-isometries acts transitively.

It has been conjectured by Gunawardena and Sparrow (see $[\mathbf{9}, \S 4.3]$ ) that the optimal upper bound for the periods of periodic points of topical functions $f: \mathbb{R}^{n} \rightarrow \mathbb{R}^{n}$ is $\left(\begin{array}{c}n \\ \lfloor n / 2\rfloor\end{array}\right)$. To see that the upper bound cannot be sharper they constructed the following example (compare [10, §2.1.3]). Put $m=\left(\begin{array}{c}n \\ \lfloor n / 2\rfloor\end{array}\right)$ and let $U$ be the collection of $m$ points $u \in\{0,1\}^{n}$ with $\sum_{i} u_{i}=\lfloor n / 2\rfloor$. Label the points in $U$ by $u^{1}, u^{2}, \ldots, u^{m}$ and put $u^{m+1}=u^{1}$. Now, define a map $f: \mathbb{R}^{n} \rightarrow \mathbb{R}^{n}$ by

$$
f(x)_{i}=\max _{k: u_{i}^{k+1}=1}\left(\min _{j: u_{j}^{k}=1}\left\{x_{j}\right\}\right) \text { for } 1 \leq i \leq n \text { and } x \in \mathbb{R}^{n} .
$$

It is easy to verify that $f$ is a topical function and that $f\left(u^{k}\right)=u^{k+1}$ for all $1 \leq k \leq m$.

In order to prove the conjecture of Gunawardena and Sparrow we show the following theorem in $\S 5$.

THEOREM 4.2. If $X$ is a finite set of incomparable elements in $\mathbb{R}^{n}$ on which an Abelian group of t-isometries acts transitively, then $|X| \leq\left(\begin{array}{c}n \\ \lfloor n / 2\rfloor\end{array}\right)$.

To establish this theorem, we apply similar ideas to the ones used in the proof of Theorem 2.1. In particular, we use the notion of a $t$-additive chain. A sequence of points $x^{1}, x^{2}, \ldots, x^{m}$ in $\mathbb{R}^{n}$ is called a $t$-additive chain if

$$
t\left(x^{1}-x^{m}\right)=\sum_{k=1}^{m-1} t\left(x^{k}-x^{k+1}\right) .
$$

The length of a $t$-additive chain is the number of distinct points in it. Note that if $a^{1}, a^{2}, \ldots, a^{m}$ is a $t$-additive chain, then $a^{m}, a^{m-1}, \ldots, a^{1}$ need not be a $t$-additive chain, because $t(x) \neq t(-x)$ in general.

We now collect several preliminary results for the proof of Theorem 4.2, beginning with two simple observations concerning $t$-additive chains.

LEMMA 4.3. If $t(x-y)=x_{i}-y_{i}$ and $t(y-z)=y_{i}-z_{i}$, then $t(x-z)=x_{i}-z_{i}$ and $x, y, z$ is a $t$-additive chain.

Proof. Note that

$$
x_{i}-z_{i} \leq t(x-z) \leq t(x-y)+t(y-z)=x_{i}-y_{i}+y_{i}-z_{i}=x_{i}-z_{i},
$$

so that $t(x-z)=x_{i}-z_{i}$ and $t(x-z)=t(x-y)+t(y-z)$.

LEMMA 4.4. If $x^{1}, x^{2}, \ldots, x^{m}$ is a t-additive chain and $t\left(x^{1}-x^{m}\right)=x_{i}^{1}-x_{i}^{m}$, then $t\left(x^{k}-x^{k+1}\right)=x_{i}^{k}-x_{i}^{k+1}$ for all $1 \leq k<m$. 
Proof. We have that

$$
t\left(x^{1}-x^{m}\right)=\sum_{k=1}^{m-1} t\left(x^{k}-x^{k+1}\right) \geq \sum_{k=1}^{m-1} x_{i}^{k}-x_{i}^{k+1}=x_{i}^{1}-x_{i}^{m}=t\left(x^{1}-x^{m}\right) .
$$

This implies that $t\left(x^{k}-x^{k+1}\right)=x_{i}^{k}-x_{i}^{k+1}$ for all $1 \leq k<m$.

Completely analogous to Lemma 2.2 one can show that transitive actions of Abelian groups of $t$-isometries satisfy the following property.

LEMMA 4.5. If $X$ is a set in $\mathbb{R}^{n}$ on which an Abelian group $\Gamma$ of t-isometries acts transitively, then for each $f \in \Gamma$ there exists a constant $d(f)$ such that $t(x-f(x))=d(f)$ for all $x \in X$.

In the same fashion as Corollary 2.3 we deduce the following consequence.

COROLlary 4.6. Let $X$ be a set in $\mathbb{R}^{n}$ on which an Abelian group $\Gamma$ of $t$-isometries acts transitively. If $x, y, z$ is a $t$-additive chain in $X$ and $f \in \Gamma$ is such that $f(x)=y$, then $y, z, f(z)$ is a $t$-additive chain in $X$.

Proof. Let $g \in \Gamma$ be such that $g(y)=z$. Using Lemma 4.5 and the fact that $\Gamma$ is Abelian, we find that

$$
\begin{aligned}
t(y-f(z)) & =t(y-g(f(y)))=t(x-g(f(x))) \\
& =t(x-y)+t(y-z)=t(z-f(z))+t(y-z) .
\end{aligned}
$$

Next, we introduce the notion of $t$-extreme pair. An ordered pair $(x, y)$ in $X \times X$, with $x \neq y$, is called $t$-extreme in $X$ if there exists no $z \in X$ such that $x, y, z$ or $z, x, y$ is a $t$-additive chain of length 3 . Note that $(x, y)$ is a $t$-extreme pair need not imply that $(y, x)$ is a $t$-extreme pair. Therefore, we consider ordered pairs instead of unordered pairs. By using Corollary 4.6 we can prove the following lemma for $t$-extreme pairs. This lemma resembles Lemma 2.4 and the proofs are quite similar.

LEMMA 4.7. If $X$ is a finite set of incomparable elements in $\mathbb{R}^{n}$ on which an Abelian group $\Gamma$ of t-isometries acts transitively, then $(x, y)$ in $X \times X$ with $x \neq y$ is $t$-extreme in $X$ if and only if there exists no $z \in X$ such that $x, y, z$ is a $t$-additive chain of length 3.

Proof. It follows from the definition that if $(x, y)$ is $t$-extreme, then there exists no $z \in X$ such that $x, y, z$ is a $t$-additive chain of length 3 . For the other implication, we assume that $(x, y)$ is not $t$-extreme. Hence, there exists $z \in X$ such that either $x, y, z$ or $z, x, y$ is a $t$-additive chain of length 3 . In the first case, we are done. In the second case, it follows from Corollary 4.6 that $x, y, f(y)$ is a $t$-additive chain, where $f \in \Gamma$ is such that $f(z)=x$. To see that it has length 3 we remark that $t(u-v)>0$ for all $u \neq v$ in $X$, as no two elements in $X$ are comparable. Thus, $t(y-f(y))=t(z-x)>0$, because $x \neq z$, and $t(x-f(y))=t(z-y)>0$, as $y \neq z$. This completes the proof. 
5. Proof of the second main result

By using the same type of arguments as in the proof of Theorem 2.1 we now prove Theorem 4.2. An important ingredient in the proof is a classic result in combinatorics, namely Sperner's theorem, which asserts the following (compare [6, Ch. 7]).

THEOREM 5.1. (Sperner) If $\mathcal{A}$ is a collection of subsets of $\{1, \ldots, n\}$ such that there are no distinct $A, B \in \mathcal{A}$ with $A \subset B$, then $|\mathcal{A}| \leq\left(\begin{array}{c}n \\ \lfloor n / 2\rfloor\end{array}\right)$.

More precisely, we use the following equivalent formulation: if $\mathcal{A}$ is an anti-chain in the partially ordered set $\left(\{0,1\}^{n}, \leq\right)$, then $|\mathcal{A}| \leq\left(\begin{array}{c}n \\ \lfloor n / 2\rfloor\end{array}\right)$.

Proof of Theorem 4.2. Let $X$ be a finite set of incomparable points in $\mathbb{R}^{n}$ on which an Abelian group $\Gamma$ of $t$-isometries acts transitively. Define a map $c: X \rightarrow\{0,1\}^{n}$ by

$$
c(x)_{i}= \begin{cases}1 & \text { if there exists } y \in X \text { such that }(x, y) \text { is } t \text {-extreme in } X \\ & \text { and } t(x-y)=x_{i}-y_{i} \\ 0 & \text { otherwise, }\end{cases}
$$

for $1 \leq i \leq n$ and $x \in X$. By Sperner's theorem it suffices to show that $c(x)$ and $c(y)$ are incomparable for all $x \neq y$ in $X$. So, suppose that $x \neq y$ in $X$. Let $z^{1}, z^{2}, \ldots, z^{m}$ be a $t$-additive chain in $X$ consisting of $m$ distinct elements, where $z^{1}=x, z^{2}=y$, and $m$ is maximal. We claim that $\left(z^{1}, z^{m}\right)$ is $t$-extreme in $X$. Indeed, if we suppose, by way of contradiction, that $\left(z^{1}, z^{m}\right)$ is not $t$-extreme, then by Lemma 4.7 there exists $u \in X$ such that $z^{1}, z^{m}, u$ is a $t$-additive chain of length 3 . This implies that $z^{1}, z^{2}, \ldots, z^{m}, u$ is a $t$-additive chain. To see that it consists of $m+1$ distinct elements we use the fact that $X$ contains no comparable elements. If $u=z^{k}$, then $k \neq m$, and moreover

$t\left(z^{1}-u\right)=t\left(z^{1}-z^{k}\right)+t\left(z^{k}-z^{m}\right)+t\left(z^{m}-u\right)=t\left(z^{1}-u\right)+t\left(u-z^{m}\right)+t\left(z^{m}-u\right)$.

This implies that $t\left(u-z^{m}\right)+t\left(z^{m}-u\right)=0$. But this contradicts the fact that $t\left(u-z^{m}\right)>0$ and $t\left(z^{m}-u\right)>0$, as $u$ and $z^{m}$ are not comparable. Thus, $z^{1}, z^{2}, \ldots, z^{m}, u$ is a $t$-additive chain with $m+1$ distinct points, which contradicts the maximality of $m$. Therefore, $\left(z^{1}, z^{m}\right)$ is $t$-extreme in $X$.

Clearly, there exists $1 \leq i \leq n$ such that $t\left(z^{1}-z^{m}\right)=z_{i}^{1}-z_{i}^{m}$, and hence the definition of $c: X \rightarrow\{0,1\}^{n}$ gives $c(x)_{i}=1$. We claim that $c(y)_{i} \neq 1$. Indeed, if $c(y)_{i}=1$, there exists $v \in X$ such that $(y, v)$ is $t$-extreme and $t(y-v)=y_{i}-v_{i}$. As $t\left(z^{1}-z^{m}\right)=z_{i}^{1}-z_{i}^{m}$, it follows from Lemma 4.4 that $t(x-y)=x_{i}-y_{i}$. Using Lemma 4.3, we find that $x, y, v$ is a $t$-additive chain. This additive chain has length 3 , because $x=v$ implies $y_{i}-x_{i}=y_{i}-v_{i}=t(y-v)=t(y-x)>0$, which contradicts the fact that $x_{i}-y_{i}=t(x-y)>0$. But $x, y, v$ cannot be a $t$-additive chain of length 3 , as $(y, v)$ is $t$-extreme. Thus, $c(y)_{i} \neq 1$ and hence $c(y)_{i}=0$. By interchanging the roles of $x$ and $y$, we also find $j$ such that $c(y)_{j}=1$ and $c(x)_{j}=0$. Therefore, $c(x)$ and $c(y)$ are not comparable, and we are done.

The result for the periods of periodic points of topical functions has consequences for the dynamics of certain cone maps. Let $\mathbb{R}_{+}^{n}$ be the standard positive cone in $\mathbb{R}^{n}$, so, $\mathbb{R}_{+}^{n}=\left\{x \in \mathbb{R}^{n} \mid x_{i} \geq 0\right.$ for all $\left.1 \leq i \leq n\right\}$ and let int $\left(\mathbb{R}_{+}^{n}\right)$ denote its interior. 
We observe that there exists a homeomorphism $L: \operatorname{int}\left(\mathbb{R}_{+}^{n}\right) \rightarrow \mathbb{R}^{n}$ given by $L(x)_{i}=$ $\log \left(x_{i}\right)$ for $1 \leq i \leq n$ and $x \in \operatorname{int}\left(\mathbb{R}_{+}^{n}\right)$. The inverse $E: \mathbb{R}^{n} \rightarrow \operatorname{int}\left(\mathbb{R}_{+}^{n}\right)$ of $L$ is, of course, given by $E(x)_{i}=e^{x_{i}}$ for $1 \leq i \leq n$ and $x \in \mathbb{R}^{n}$. As both $z \rightarrow \log (z)$ and $z \rightarrow e^{z}$ are monotone functions, we have that if $g: \operatorname{int}\left(\mathbb{R}_{+}^{n}\right) \rightarrow \operatorname{int}\left(\mathbb{R}_{+}^{n}\right)$ is order-preserving, then $f: \mathbb{R}^{n} \rightarrow \mathbb{R}^{n}$, given by $f(x)=L(g(E(x)))$ for $x \in \mathbb{R}^{n}$, is also order-preserving. Moreover, it is easy to see that if $g: \operatorname{int}\left(\mathbb{R}_{+}^{n}\right) \rightarrow \operatorname{int}\left(\mathbb{R}_{+}^{n}\right)$ is homogeneous, i.e. $g(\lambda x)=$ $\lambda g(x)$ for all $x \in \operatorname{int}\left(\mathbb{R}_{+}^{n}\right)$ and $\lambda>0$, then $f$ is additively homogeneous. Conversely, one can show that if $f: \mathbb{R}^{n} \rightarrow \mathbb{R}^{n}$ is a topical function, then $g: \operatorname{int}\left(\mathbb{R}_{+}^{n}\right) \rightarrow \operatorname{int}\left(\mathbb{R}_{+}^{n}\right)$, given by $g(x)=E(f(L(x)))$ for all $x \in \operatorname{int}\left(\mathbb{R}_{+}^{n}\right)$, is order-preserving and homogeneous. Therefore, order-preserving homogeneous maps $g: \operatorname{int}\left(\mathbb{R}_{+}^{n}\right) \rightarrow \operatorname{int}\left(\mathbb{R}_{+}^{n}\right)$ have the same dynamical behaviour as topical functions $f: \mathbb{R}^{n} \rightarrow \mathbb{R}^{n}$. Consequently, the following assertion is true.

THEOREM 5.2. The optimal upper bound for the periods of periodic points of maps $g: \operatorname{int}\left(\mathbb{R}_{+}^{n}\right) \rightarrow \operatorname{int}\left(\mathbb{R}_{+}^{n}\right)$ that are order-preserving and homogeneous is $\left(\begin{array}{c}n \\ \lfloor n / 2\rfloor\end{array}\right)$.

We conclude the paper by indicating a connection between the two main results. To every sup-norm non-expansive map $f: M \rightarrow M$, where $M \subset \mathbb{R}^{n}$, a $t$-non-expansive map $g: M^{\prime} \rightarrow M^{\prime}$, where $M^{\prime} \subset \mathbb{R}^{2 n}$, can be associated such that $g$ has the same dynamical behaviour as $f$. Indeed, if we let $\tau: \mathbb{R}^{n} \rightarrow \mathbb{R}^{2 n}$ be given by $\tau(x)=(x,-x)$ for all $x \in \mathbb{R}^{n}$, then

$$
t(\tau(x)-\tau(y))=\max _{1 \leq i \leq 2 n}\left\{\tau(x)_{i}-\tau(y)_{i}\right\}=\max \{t(x-y), t(y-x)\}=\|x-y\|_{\infty} .
$$

This implies that if $f$ is sup-norm non-expansive, then $g=\tau \circ f \circ \tau^{-1}$ is $t$-nonexpansive. In particular, if $\Gamma$ is an Abelian group of sup-norm isometries $f: X \rightarrow X$ that acts transitively on $X$ and $Y=\tau(X)$, then $Y$ contains no comparable elements and $\Gamma^{\prime}=\left\{\tau \circ f \circ \tau^{-1}: Y \rightarrow Y \mid f \in \Gamma\right\}$ is an Abelian group of $t$-isometries that acts transitively on $Y$. By applying Theorem 4.2, we immediately deduce that each finite set $X$ in $\mathbb{R}^{n}$, on which an Abelian group of sup-norm isometries acts transitively, has at most $\left(\begin{array}{c}2 n \\ n\end{array}\right)$ elements. From Stirling's formula it follows that $\left(\begin{array}{c}2 n \\ n\end{array}\right) \sim 4^{n} / \sqrt{\pi n}$, which is a worse upper bound than the one in Theorem 2.1.

Acknowledgements. The authors are very grateful to Roger Nussbaum for his comments on an earlier version of the paper. The first author was supported by a TALENT-Fellowship of the Netherlands Organization for Scientific Research (NWO).

\section{REFERENCES}

[1] M. A. Akcoglu and U. Krengel. Nonlinear models of diffusion on a finite space. Probab. Theory Related Fields 76(4) (1987), 411-420.

[2] M. Akian and S. Gaubert. Spectral theorem for convex monotone homogeneous maps, and ergodic control. Nonlinear Anal. 52(2) (2003), 637-679.

[3] F. Baccelli, G. Cohen, G. J. Olsder and J. P. Quadrat. Synchronization and Linearity: An Algebra for Discrete Event Systems (Wiley Series on Probability and Mathematical Statistics). Wiley, Chichester, 1992. 
[4] A. Blokhuis and H. A. Wilbrink. Alternative proof of Sine's theorem on the size of a regular polygon in $\mathbb{R}^{k}$ with the $\ell_{\infty}$-metric. Discrete Comput. Geom. 7(4) (1992), 433-434.

[5] T. Bousch and J. Mairesse. Asymptotic height optimization for topical IFS, Tetris heaps, and the finiteness conjecture. J. Amer. Math. Soc. 15(1) (2002), 77-111.

[6] P. J. Cameron. Combinatorics: Topics, Techniques, Algorithms. Cambridge University Press, Cambridge, 1994.

[7] M. G. Crandall and L. Tartar. Some relations between nonexpansive and order preserving mappings. Proc. Amer. Math. Soc. 78(3) (1980), 385-390.

[8] S. Gaubert and J. Gunawardena. The Perron-Frobenius theory for homogeneous, monotone functions. Trans. Amer. Math. Soc. 356(12) (2004), 4931-4950.

[9] J. Gunawardena. An introduction to idempotency. Idempotency (Publications of the Newton Institute, 11). Ed. J. Gunawardena. Cambridge University Press, Cambridge, 1998, pp. 1-49.

[10] J. Gunawardena. From max-plus algebra to nonexpansive mappings: a nonlinear theory for discrete event systems. Theoret. Comput. Sci. 293(1) (2003), 141-167.

[11] J. Gunawardena and M. S. Keane. On the existence of cycle times for some nonexpansive maps. Technical Report HPL-BRIMS-95-003, Hewlett-Packard Laboratories, 1995.

[12] B. Lemmens, R. D. Nussbaum and S. M. Verduyn Lunel. Lower and upper bounds for $\omega$-limit sets of nonexpansive maps. Indag. Math. (N.S.) 12(2) (2001), 191-211.

[13] B. Lemmens and M. Scheutzow. A characterization of the periods of periodic points of 1-norm nonexpansive maps. Selecta Math. (N.S.) 9(4) (2003), 557-578.

[14] S.-K. Lo. Abschätzungen für starre Mengen in $\mathbb{R}^{l}$ mit $\ell_{\infty}$-oder polygonaler Norm. Diplomarbeit, Georg-August-Universität, Göttingen, 1989.

[15] R. Lyons and R. D. Nussbaum. On transitive and commutative finite groups of isometries. Fixed Point Theory and Applications. Ed. K.-K. Tan. World Scientific, Singapore, 1992, pp. 189-228.

[16] P. Martus. Asymptotic properties of nonstationary operator sequences in the nonlinear case. PhD Thesis, Friedrich-Alexander University, Erlangen-Nürnberg, Germany, 1989.

[17] M. Misiurewicz. Rigid sets in finite dimensional $l_{1}$-spaces. Technical Report 45, Mathematica Göttingensis Schriftenreihe des Sonderforschungsbereichs Geometrie und Analysis, 1987.

[18] R. D. Nussbaum. Omega limit sets of nonexpansive maps: finiteness and cardinality estimates. Differential Integral Equations 3(3) (1990), 523-540.

[19] R. D. Nussbaum. Hilbert's projective metric and iterated nonlinear maps. Mem. Amer. Math. Soc. 75(391) (1988), 1-137.

[20] R. D. Nussbaum. Iterated nonlinear maps and Hilbert's projective metric II. Mem. Amer. Math. Soc. 79(401) (1989), 1-118.

[21] R. D. Nussbaum and M. Scheutzow. Admissible arrays and a generalization of Perron-Frobenius theory. J. London Math. Soc. 58(2) (1998), 526-544.

[22] R. D. Nussbaum, M. Scheutzow and S. M. Verduyn Lunel. Periodic points of nonexpansive maps and nonlinear generalizations of the Perron-Frobenius theory. Selecta Math. (N.S.) 4(1) (1998), 1-41.

[23] R. D. Nussbaum and S. M. Verduyn Lunel. Asymptotic estimates for the periods of periodic points of non-expansive maps. Ergod. Th. \& Dynam. Sys. 23 (2003), 1199-1226.

[24] M. Scheutzow. Periods of nonexpansive operators on finite $l_{1}$-spaces. European J. Combin. 9 (1988), 73-78.

[25] R. Sine. A nonlinear Perron-Frobenius theorem. Proc. Amer. Math. Soc. 109(2) (1990), 331-336.

[26] D. Weller. Hilbert's metric, part metric and self mappings of a cone. PhD Thesis, Universität Bremen, Germany, 1987. 